\title{
Three-Dimensional Reciprocal Invisibility Cloak with Multilayered Structure
}

\author{
Yanglu Xuan $^{1}$ (D), Xinhe $\mathrm{Xu}^{1}$ \\ ${ }^{1}$ School of Information Engineering, Nanchang Hangkong University, Nanchang, China \\ YangluXuan(2623822646@qq.com); Xinhe Xu (xxh_rifc@hotmail.com)
}

\begin{abstract}
We investigate a three-dimensional (3D) reciprocal invisibility cloak with a multilayered structure based on coordinate transformation theory and complementary media. There has been considerable research on invisibility cloaks such as the external cloak, which requires complex "anti-objects", and closed cloaks of different shapes, in which the object enclosed is blind. Because all these are hard to realize in actual application, we apply a uniform layer to the whole construct into several parts in efforts to replace the ideal cloak with the aim to reduce complexity in actual fabrication. This multilayered cloak allows exchange of information without requiring "anti-objects". All results were simulated and calculated in the Cartesian coordinate system and with the Comsol Multiphysics software. Electrical field simulations verified the efficiency of the designed cloak and the computed radar cross section (RCS) to illustrate the validity of this method and its efficiency in replacing the ideal cloak.
\end{abstract}

Index Terms - Complementary media, Coordinate transformation theory, Multilayered structure, Radar cross section (RCS).

\section{INTRODUCTION}

The concept of an invisibility cloak based on transformation optics and the coordinate transformation theory was first proposed by Pendry et al. [1]. Schurig et al. [2] demonstrated the theory behind the cloak according to a ray-tracing program, which allowed them to construct a cylindrical invisibility cloak operating in the microwave frequency band. This study has inspired many different types of cloaks, including wave rotators [3], elliptic cloaks [4]-[7], cloaks with arbitrary shape [8]-[10], electromagnetic wave transparent devices [11], and electromagnetic concentrators [12]. However, all of these cases present a closed region, such that the object enclosed within the cloak is blind, i.e., it cannot communicate with the outside world. This called for an investigation of a new type of invisibility cloak, which enables information exchange between the inside and outside of the cloak. Ma et al. [13] presented an open cloak that realizes this communication, but it was achieved at the cost of perfect invisibility. Moreover, Lai et al. [14] proposed a new design, namely, the external invisibility cloak, based on complementary media. This cloak can ensure that the object outside the cloak is not only hidden, but that it can also communicate with the outside world. However, the key to realizing invisibility depends on some specific "anti- 
objects" embedded inside the cloak. Thus, Luo et al. [15] proposed an invisible cloak with multiple regions. The object in the external cavity and internal space of one cloak can be concealed simultaneously, but it still requires "anti-objects". In addition, Yang et al. [16] investigated an interior invisible cloak that combines communication ability and invisibility. They also presented another external invisible cloak with an arbitrary cross section [17]. Recently, several cloaks have been proposed [18]-[21]. However, all of the above-mentioned cases describe two-dimensional (2D) cloaks, which are easy to implement and simulate but inconvenient in practical applications, as the incident waves have unforeseeable angles.

We propose a uniform layered method for realizing a three-dimensional (3D) reciprocal invisibility cloak that differs from the previous designs. In our design, a 3D spherical structure solves the problem of the incident wave with different directions. Moreover, this invisibility cloak does not require any "anti-objects" and is capable of communicating with the outside world while maintaining a good invisibility performance. In particular, the significance of this proposed design is that the multilayered cloak is capable of replacing the ideal cloak as a means of reducing the complexity in actual application. This is because consistency cannot be guaranteed in material parameters of a large region, but division of the region into several tiny sections can ensure that the parameters of the same section are consistent, thereby facilitating the application. Moreover, the layered design is helpful for reducing scattering from the object. Researchers at Duke University used a 10-layered split-ring resonator to approximate the ideal closed cloak in their invisibility cloak design [2]. Based on this idea and the coordinate transformation theory, we derive the parameter expressions for each region. Finally, we implement full-wave simulations by Comsol Multiphysics to verify the validity and performance of this design.

\section{CLOAK THEORY AND DESIGN}

A schematic of the xoy cross section for the 3D reciprocal invisible cloak is shown in Fig. 1. It comprises three regions: a uniform layered cloak (region I), complementary media (region II), and a concealed zone (region III). The radii of the circles from inside to outside are $a, b$, and $c$, respectively. The point in region III is expressed by coordinates $(x, y, z)$; the point in region II, by $\left(x^{\prime}, y^{\prime}, z^{\prime}\right)$; and the point in region I, by $\left(x^{\prime \prime}, y^{\prime \prime}, z^{\prime \prime}\right)$. 


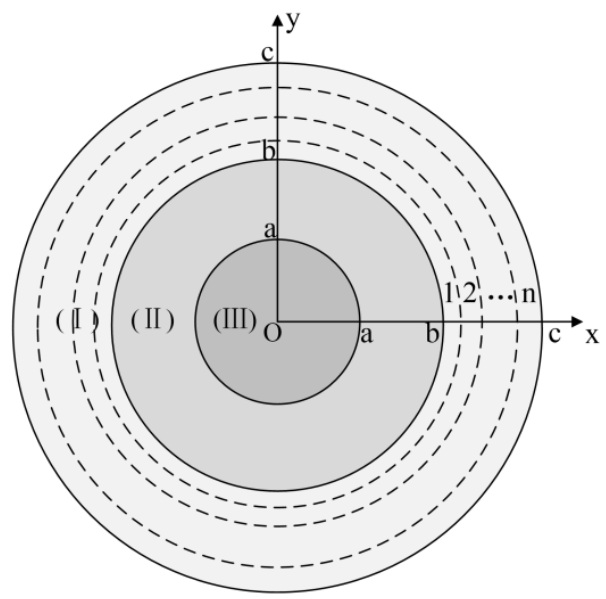

Fig. 1. Schematic of the xoy cross-section for the 3D reciprocal invisible cloak ( $n=1,2 \ldots 10$ ).

The main purpose of our design is that an object in the region $0 \leq r \leq a$ can be hidden by the invisibility cloak in the region $b \leq r$ " $\leq c$, and that the invisibility can be "cancelled" by the complementary media in the region $a \leq r^{\prime} \leq b$. Thus, the design we proposed can be summarized in two steps. First, we compress region III $(0 \leq r \leq a)$ into region II $\left(a \leq r^{\prime} \leq b\right)$ [22]. Second, the entire region $(0 \leq r \leq c)$ is transformed into region $\mathrm{I}\left(b \leq r^{\prime \prime} \leq c\right)$.

The transformation expression of the first step is as follows:

$$
r^{\prime}=b+\left(\frac{a-b}{a}\right) r, \quad \theta^{\prime}=\theta, \quad \phi^{\prime}=\phi .
$$

where $a$ is the radius of the inner sphere, $b$ is the radius of the middle sphere, $r=\sqrt{x^{2}+y^{2}+z^{2}}$, $\theta=\arccos \left(\frac{z}{r}\right)$, and $\phi=\arctan \left(\frac{y}{x}\right)$

Applying the spherical coordinate system $(r, \theta, \phi)$, we can derive the Jacobian transformation matrix as follows:

$$
\Lambda^{\prime}=\left(\begin{array}{ccc}
\frac{\partial r^{\prime}}{\partial r} & \frac{1}{r} \frac{\partial r^{\prime}}{\partial \theta} & \frac{1}{r \sin (\theta)} \frac{\partial r^{\prime}}{\partial \phi} \\
r^{\prime} \frac{\partial \theta^{\prime}}{\partial r} & \frac{r^{\prime}}{r} \frac{\partial \theta^{\prime}}{\partial \theta} & \frac{r^{\prime}}{r \sin (\theta)} \frac{\partial \theta^{\prime}}{\partial \phi} \\
r^{\prime} \sin \left(\theta^{\prime}\right) \frac{\partial \phi^{\prime}}{\partial r} & \frac{r^{\prime} \sin \left(\theta^{\prime}\right)}{r} \frac{\partial \phi^{\prime}}{\partial \theta} & \frac{r^{\prime} \sin \left(\theta^{\prime}\right)}{r \sin (\theta)} \frac{\partial \phi^{\prime}}{\partial \phi}
\end{array}\right)
$$

Drawing on the coordinate transformation theory and the invariant form of Maxwell's equations, we can obtain permittivity and permeability tensors in the complementary media:

$$
\varepsilon^{\prime}=\frac{\Lambda^{\prime} \cdot \Lambda^{\prime T}}{\operatorname{det}\left(\Lambda^{\prime}\right)} \cdot \varepsilon, \quad \mu^{\prime}=\frac{\Lambda^{\prime} \cdot \Lambda^{\prime T}}{\operatorname{det}\left(\Lambda^{\prime}\right)} \cdot \mu .
$$

where $\Lambda^{\prime}$ is the Jacobian transformation matrix, while $\varepsilon^{\prime}, \mu^{\prime}$ and $\varepsilon, \mu$ are the permittivity and permeability tensors of regions II and III, respectively.

Region III is free space, such that $\varepsilon=\varepsilon_{0}, \mu=\mu_{0}$. Next, we acquire the permittivity and permeability tensors of region II in the spherical coordinate system: 


$$
\varepsilon^{\prime}=\mu^{\prime}=\left(\begin{array}{ccc}
\varepsilon_{r r}^{\prime} & 0 & 0 \\
0 & \varepsilon_{\theta \theta}^{\prime} & 0 \\
0 & 0 & \varepsilon_{\phi \phi}^{\prime}
\end{array}\right)
$$

The commercial software Comsol Multiphysics is based on Cartesian coordinates; therefore, we employ the relationship between the spherical $\left(r^{\prime}, \theta^{\prime}, \phi^{\prime}\right)$ and Cartesian coordinates $\left(x^{\prime}, y^{\prime}, z^{\prime}\right)$, to express the tensors of region II as follows:

$$
\varepsilon^{\prime}=\mu^{\prime}=\left(\begin{array}{ccc}
\varepsilon_{x x}^{\prime} & \varepsilon_{x y}^{\prime} & \varepsilon_{x z}^{\prime} \\
\varepsilon_{y x}^{\prime} & \varepsilon_{y y}^{\prime} & \varepsilon_{y z}^{\prime} \\
\varepsilon_{z x}^{\prime} & \varepsilon_{z y}^{\prime} & \varepsilon_{z z}^{\prime}
\end{array}\right)
$$

in which,

$$
\begin{gathered}
\varepsilon_{x x}^{\prime}=\mu_{x x}^{\prime}=\frac{\sin ^{2}(\theta) \cos ^{2}(\phi)\left(r^{\prime}-b\right)^{2}}{r^{\prime 2}} \frac{a}{a-b}+\cos ^{2}(\theta) \cos ^{2}(\phi) \frac{a}{a-b}+\sin ^{2}(\phi) \frac{a}{a-b} \\
\varepsilon_{x y}^{\prime}=\varepsilon_{y x}^{\prime}=\mu_{x y}^{\prime}=\mu_{y x}^{\prime}=\frac{\sin ^{2}(\theta) \sin (\phi) \cos (\phi)\left(r^{\prime}-b\right)^{2}}{r^{\prime 2}} \frac{a}{a-b}+\cos ^{2}(\theta) \sin (\phi) \cos (\phi) \frac{a}{a-b}-\sin (\phi) \cos (\phi) \frac{a}{a-b} \\
\varepsilon_{x z}^{\prime}=\varepsilon_{z x}^{\prime}=\mu_{x z}^{\prime}=\mu_{z x}^{\prime}=\frac{\sin (\theta) \cos (\theta) \cos (\phi)\left(r^{\prime}-b\right)^{2}}{r^{\prime 2}} \frac{a}{a-b}-\sin (\theta) \cos (\theta) \cos (\phi) \frac{a}{a-b} \\
\varepsilon_{y y}^{\prime}=\mu_{y y}^{\prime}=\frac{\sin ^{2}(\theta) \sin ^{2}(\phi)\left(r^{\prime}-b\right)^{2}}{r^{\prime 2}} \frac{a}{a-b}+\cos ^{2}(\theta) \sin ^{2}(\phi) \frac{a}{a-b}+\cos ^{2}(\phi) \frac{a}{a-b} \\
\varepsilon_{y z}^{\prime}=\varepsilon_{z y}^{\prime}=\mu_{y z}^{\prime}=\mu_{z y}^{\prime}=\frac{\sin (\theta) \cos (\theta) \sin (\phi)\left(r^{\prime}-b\right)^{2}}{r^{\prime 2}} \frac{a}{a-b}-\sin (\theta) \cos (\theta) \sin (\phi) \frac{a}{a-b} \\
\varepsilon_{z z}^{\prime}=\mu_{z z}^{\prime}=\frac{\cos ^{2}(\theta)\left(r^{\prime}-b\right)^{2}}{r^{\prime 2}} \frac{a}{a-b}+\sin ^{2}(\theta) \frac{a}{a-b}
\end{gathered}
$$

Then the transformation equation of the second step can be expressed as:

$$
r^{\prime \prime}=b+\left(\frac{c-b}{c}\right) r, \quad \theta^{\prime \prime}=\theta, \quad \phi^{\prime \prime}=\phi .
$$

Similarly, we obtain the permittivity and permeability tensors in region I, i.e., $b \leq r^{\prime \prime} \leq c$ :

$$
\varepsilon^{\prime \prime}=\frac{\Lambda^{\prime \prime} \cdot \Lambda^{\prime \prime T}}{\operatorname{det}\left(\Lambda^{\prime \prime}\right)} \cdot \varepsilon, \quad \mu^{\prime \prime}=\frac{\Lambda^{\prime \prime} \cdot \Lambda^{\prime \prime T}}{\operatorname{det}\left(\Lambda^{\prime \prime}\right)} \cdot \mu
$$

So the permittivity and permeability tensors of region I in the spherical coordinate system can be expressed:

$$
\varepsilon^{\prime \prime}=\mu^{\prime \prime}=\left(\begin{array}{ccc}
\varepsilon_{r r}^{\prime \prime} & 0 & 0 \\
0 & \varepsilon_{\theta \theta}^{\prime \prime} & 0 \\
0 & 0 & \varepsilon_{\phi \phi}^{\prime \prime}
\end{array}\right)
$$

Therefore, we can express the tensors of region I in Cartesian coordinates as follows:

where

$$
\varepsilon^{\prime \prime}=\mu^{\prime \prime}=\left(\begin{array}{lll}
\varepsilon_{x x}^{\prime \prime} & \varepsilon_{x y}^{\prime \prime} & \varepsilon_{x z}^{\prime \prime} \\
\varepsilon_{y x}^{\prime \prime} & \varepsilon_{y y}^{\prime \prime} & \varepsilon_{y z}^{\prime \prime} \\
\varepsilon_{z x}^{\prime \prime} & \varepsilon_{z y}^{\prime \prime} & \varepsilon_{z z}^{\prime \prime}
\end{array}\right)
$$

$$
\begin{gathered}
\varepsilon_{x x}^{\prime \prime}=\mu_{x x}^{\prime \prime}=-\frac{\sin ^{2}(\theta) \cos ^{2}(\phi) r^{2}}{r^{\prime 2}} \frac{b-c}{c}-\cos ^{2}(\theta) \cos ^{2}(\phi) \frac{c}{b-c}-\sin ^{2}(\phi) \frac{c}{b-c} \\
\varepsilon_{x y}^{\prime \prime}=\varepsilon_{y x}^{\prime \prime}=\mu_{x y}^{\prime \prime}=\mu_{y x}^{\prime \prime}=-\frac{\sin ^{2}(\theta) \sin (\phi) \cos (\phi) r^{2}}{r^{\prime \prime}} \frac{b-c}{c}-\cos ^{2}(\theta) \sin (\phi) \cos (\phi) \frac{c}{b-c}+\sin (\phi) \cos (\phi) \frac{c}{b-c} \\
\varepsilon_{x z}^{\prime \prime}=\varepsilon_{z x}^{\prime \prime}=\mu_{x z}^{\prime \prime}=\mu_{z x}^{\prime \prime}=-\frac{\sin (\theta) \cos (\theta) \cos (\phi) r^{2}}{r^{\prime \prime}} \frac{b-c}{c}+\sin (\theta) \cos (\theta) \cos (\phi) \frac{c}{b-c}
\end{gathered}
$$




$$
\begin{gathered}
\varepsilon_{y y}^{\prime \prime}=\mu_{y y}^{\prime \prime}=\frac{\sin ^{2}(\theta) \sin ^{2}(\phi) r^{2}}{r^{\prime 2}} \frac{b}{b-c}-\cos ^{2}(\theta) \sin ^{2}(\phi) \frac{c}{b-c}-\cos ^{2}(\phi) \frac{c}{b-c} \\
\varepsilon_{y z}^{\prime \prime}=\varepsilon_{z y}^{\prime \prime}=\mu_{y z}^{\prime \prime}=\mu_{z y}^{\prime \prime}=-\frac{\sin (\theta) \cos (\theta) \sin (\phi) r^{2}}{r^{\prime 2}} \frac{b-c}{c}+\sin (\theta) \cos (\theta) \sin (\phi) \frac{c}{b-c} \\
\varepsilon_{z z}^{\prime \prime}=\mu_{z z}^{\prime \prime}=-\frac{\cos ^{2}(\theta) r^{2}}{r^{\prime 2}} \frac{b}{b-c}-\sin ^{2}(\theta) \frac{c}{b-c}
\end{gathered}
$$

\section{SIMULATION AND DISCUSSION}

Comsol Multiphysics software was used to verify the validity of the proposed parameter expressions. A simulation with an ideal invisibility cloak was designed to provide a comparison to the uniform layered cloak. The radii of the spheres from inside to outside are $a=0.05 \mathrm{~m}, b=0.1 \mathrm{~m}$, and $c=0.2 \mathrm{~m}$, respectively. In the following simulation, the operation frequency of the multilayered cloak is set to $1.2 \mathrm{GHz}$.

In fact, the approximation of mutilayered method is that division of the region I into several tiny sections can ensure that the parameters of the same section are consistent. In other words, the constitutive parameter (the relative permittivity and permeability) in the spherical coordinate system gradually approximates towards the ideal reciprocal cloak. Therefore, the relative permittivity and permeability curves for the different layered cloaks are plotted in Fig. 2. The thickness of each layer was the same because we used a uniform approach. It can be found that the relative permittivity and permeability of the various cloaks gradually approximated towards the ideal reciprocal cloak when the number of layers increased, that is, the relative permittivity and permeability of 8-layered cloak are already almost equivalent to the ideal reciprocal cloak.

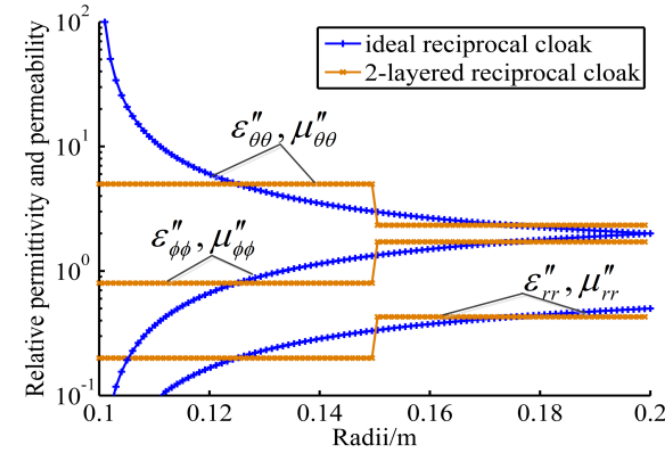

(a)

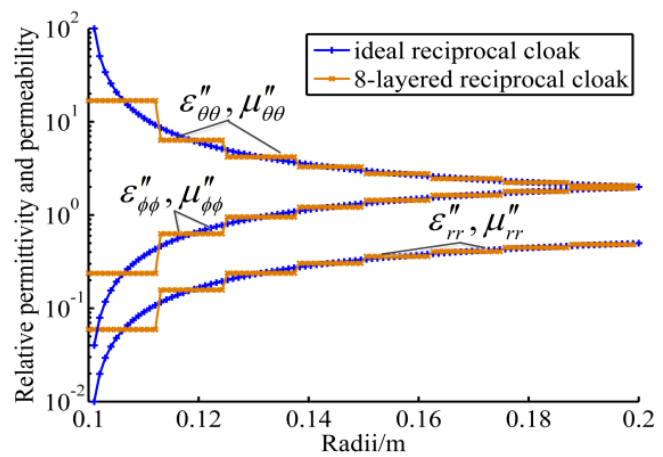

(c)

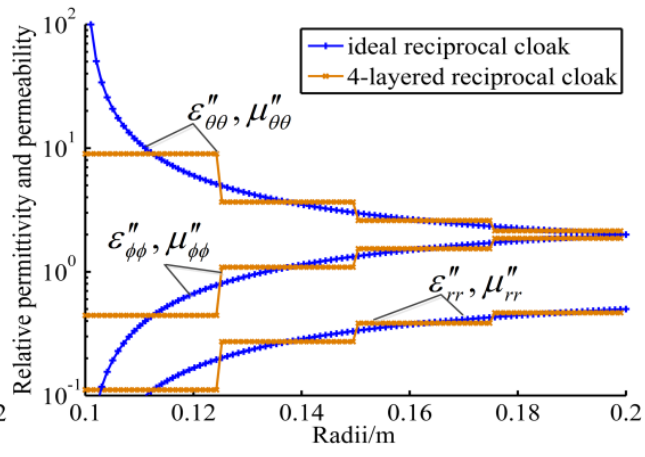

(b)

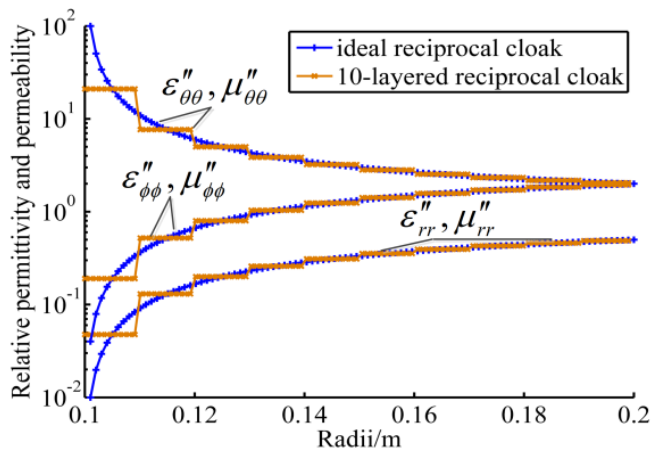

(d)

Fig. 2. The relative permittivity and permeability curves for the different layered cloaks: (a) 2-layered reciprocal cloak, (b) 4-layered reciprocal cloak, (c) 8-layered reciprocal cloak, (d) 10-layered reciprocal cloak. 
The electric $(E)$ field distribution for the ideal invisible cloak is shown in Fig. 3. The 3D $E$ field distribution is shown in Fig. 3 (a), while Fig. 3 (b) depicts the xoy 2D cross-sectional field distribution. The obtained results indicate that the electric field was able to perfectly propagate into the cloak. This demonstrates perfect invisible performance of the ideal cloak. Fig. 4 illustrates the $E$ field distribution for a 2-layered invisible cloak. From Fig. 4 (b), we can deduce that the forward scattering is quite apparent, and the wavefront becomes discontinuous. Fig. 5 illustrates the $E$ field distribution for the 4layered invisible cloak. The comparison of Figs. 5 (b) and 4 (b) indicates that the scattering is slightly reduced by the addition of two layers.

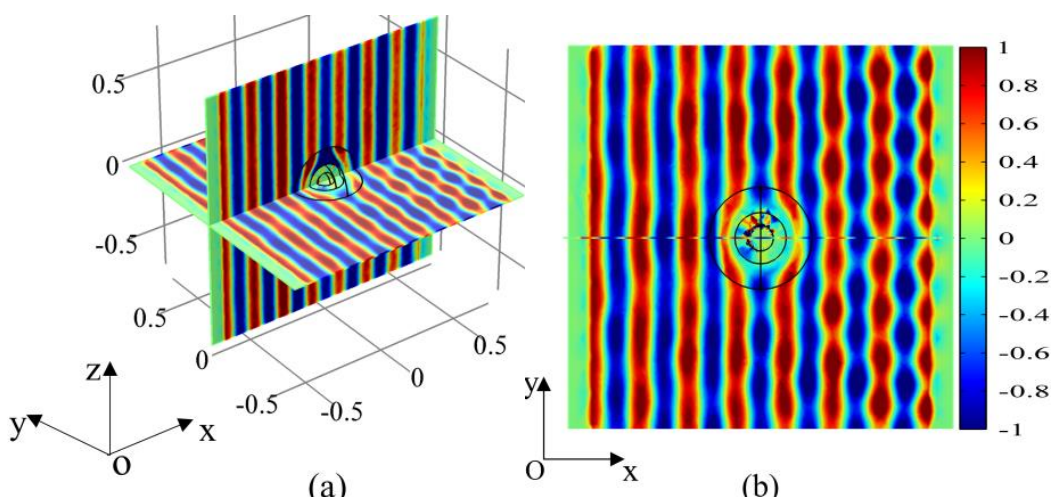

(a)

(b)

Fig. 3. Electric field distribution for the ideal invisible cloak: (a) 3D field distribution, (b) xoy cross-sectional field

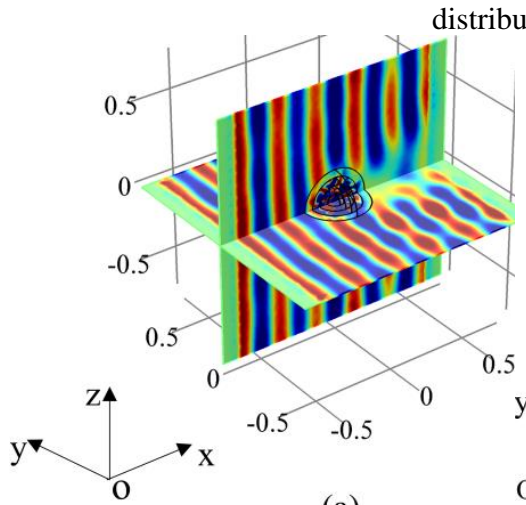

(a)

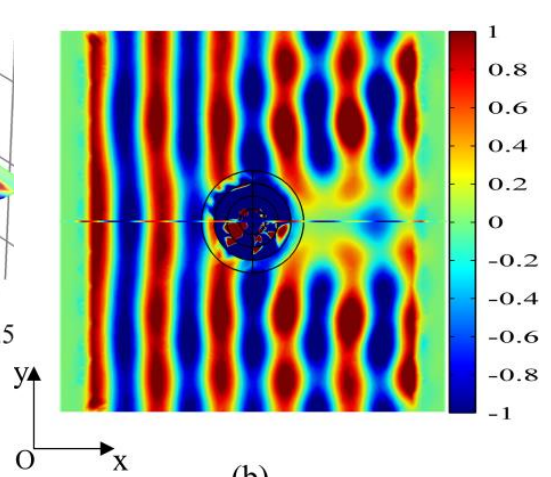

(b)

Fig. 4. Electric field distribution for the 2-layered invisible cloak: (a) 3D field distribution, (b) xoy cross-sectional field

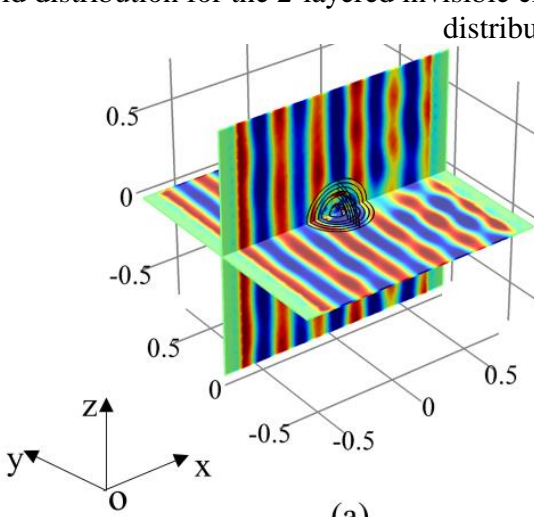

(a)

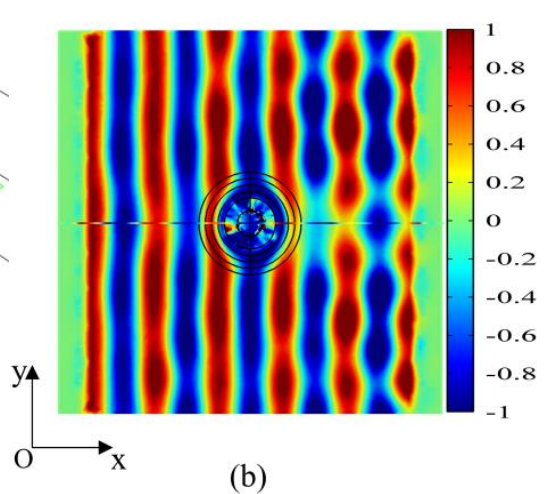

(b)

Fig. 5. Electric field distribution for the 4-layered invisible cloak: (a) 3D field distribution, (b) xoy cross-sectional field distribution.

The $E$ field distribution for the 8-layered invisible cloak is shown in Fig. 6. In Fig. 6 (b), we can see that the scattering is further reduced. Ultimately, Fig. 7 illustrates the $E$ field distribution for the 10- 
layered invisible cloak. Fig. 7 (b) portrays an electric field distribution similar to Fig. 6 (b) corresponding to the 8-layered cloak.

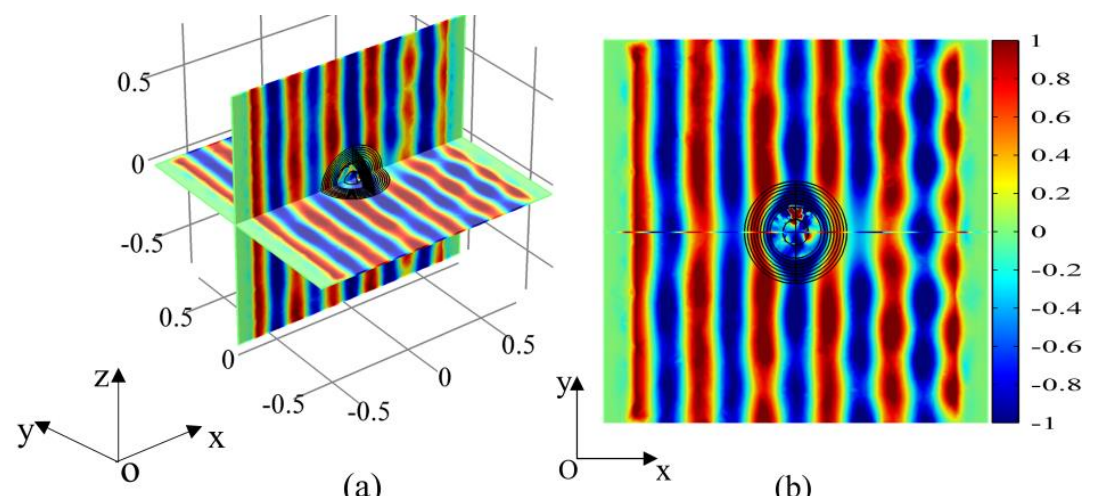

(a)

(b)

Fig. 6. Electric field distribution for the 8-layered invisible cloak: (a) 3D field distribution, (b) xoy cross-sectional field

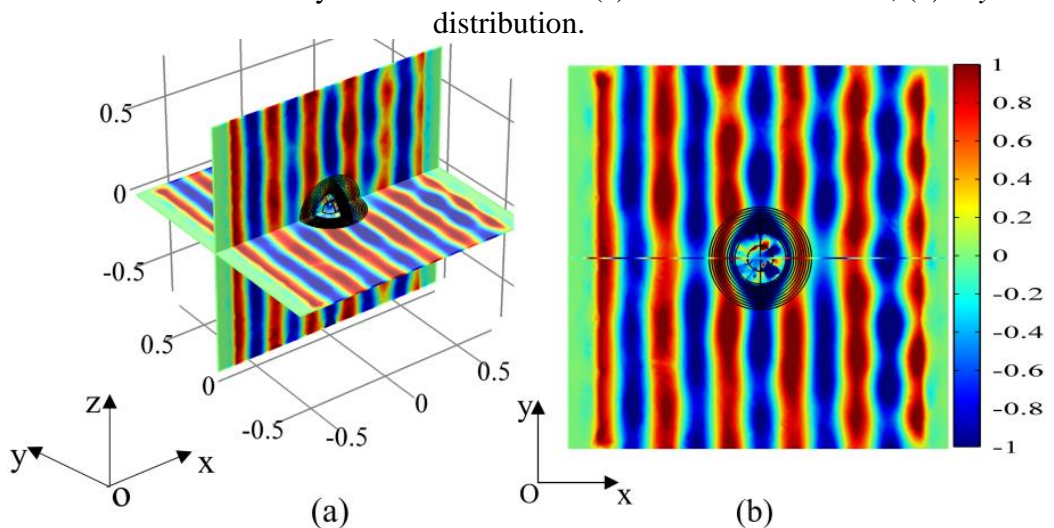

(a)

(b)

Fig. 7. Electric field distribution for the 10-layered invisible cloak: (a) 3D field distribution, (b) xoy cross-sectional field distribution.

Fig. 8 shows the near field curves for the different layered cloaks. The three curves for the 8-, 10layered, and the ideal cloak are basically coincidental, whereas the 2- and 4-layered cloak curves exhibit dramatic variations. This indicates that wave equations of the former three cases have similar solutions in the near field. The far field distribution can be measured by the RCS value, where larger RCS values imply larger scattering in the far field. The far field curves for the different layered cloaks are shown in Fig. 9. The 2-layered cloak exhibits the largest RCS values on average with significant variations, while those of the ideal cloak are the smallest and fairly constant. The remaining curves vary between these two extremes. The three curves for the far field of the 8-, 10-layered, and the ideal cloak produced the smallest RCS values. This result implies that the scattering in the far field becomes quite small when the layered number is 8 or more. Thus, the ideal cloak can be replaced by a uniform layered cloak without any negative impact on the invisibility performance. In addition, note that the reduction of the RCS value is slight when the layerd number is over 8 . When wanting to reduce the RCS value further, we must add the uniform layered number sharply, which would greatly increase the complexity of the design. Therefore, we employ the 8-layered cloak for testing of the invisibility performance and communication properties. 


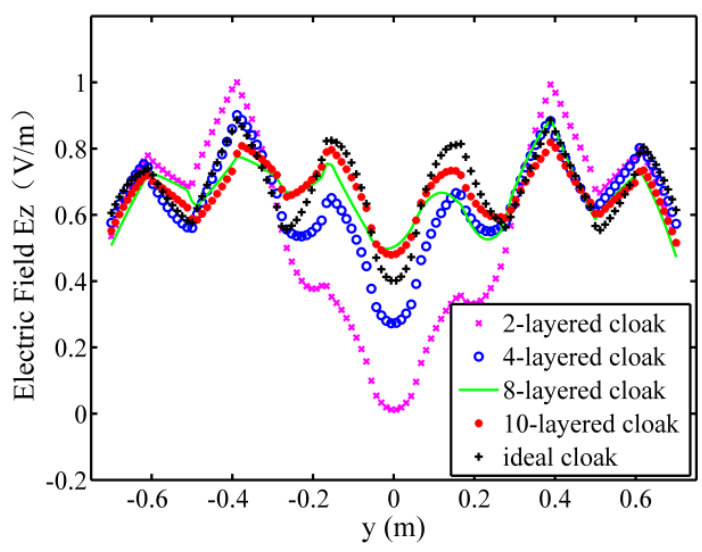

Fig. 8. Near field curves for the different layered cloaks.

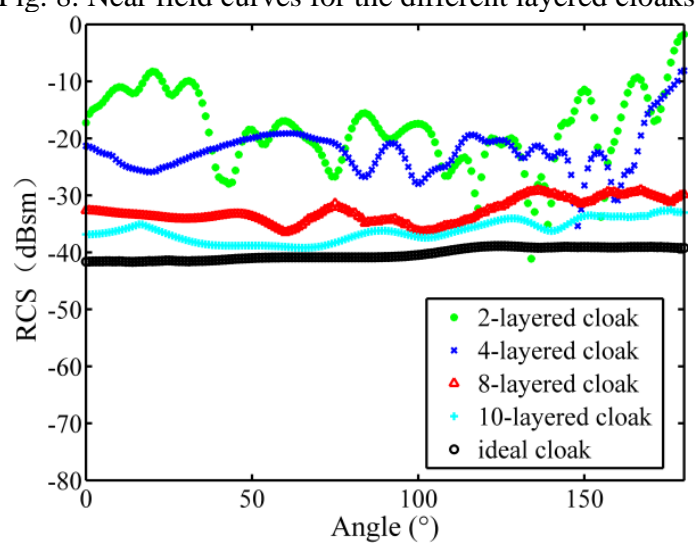

Fig. 9. Far field curves for the different layered cloaks.

In order to verify communication abilities of an object within the cloak, an ellipsoid object $\left(\varepsilon_{r}=3, \mu_{r}=1\right)$ was placed in the region $0 \leq r \leq a$. The cylindrical wave was generated by a line current located at $(-0.75 \mathrm{~m},-0.88 \mathrm{~m}, 0 \mathrm{~m})$ near the cloak. Figs. 10 (a), (b) and (c), (d) show the $E$ field distribution for the 8-layered cloak without and with an object in place, respectively. The electric field can propagate into the interior space of the cloak, and thereupon recover its original trajectory. Results show that it is possible for the hidden object to communicate with the outside world. The RCS value of the 8-layered cloak with and without the object was calculated using Matlab, and the far field curves are shown in Fig. 11. The two curves are extremely similar in shape, with approximately $3 \mathrm{~dB}$ difference within the range of $0^{\circ}-180^{\circ}$. This strongly implies that the invisibility performance and communication ability of the cloak are maintained even in the presence of an object in the interior of the cloak. This presents a significant advantage, as the complex implementation of "anti-objects" is not required. 


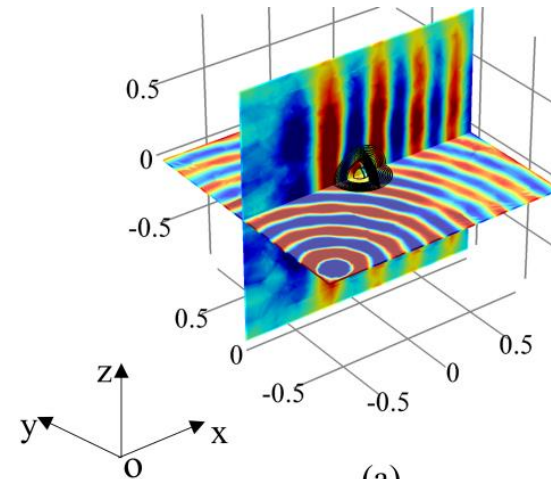

(a)

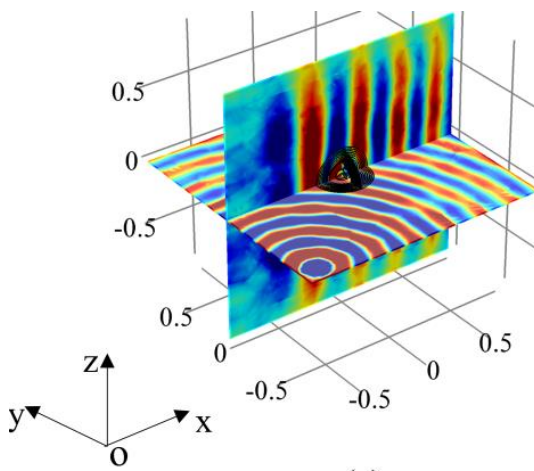

(c)

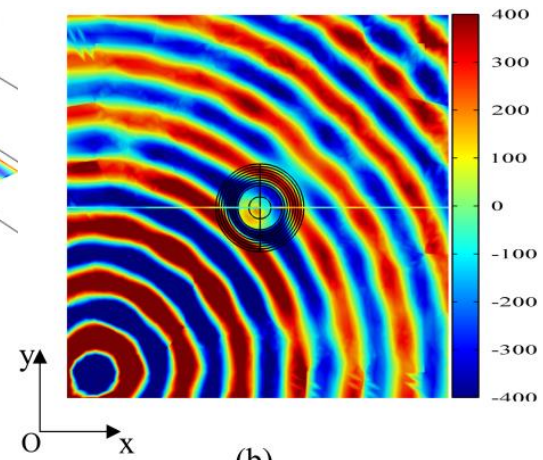

(b)

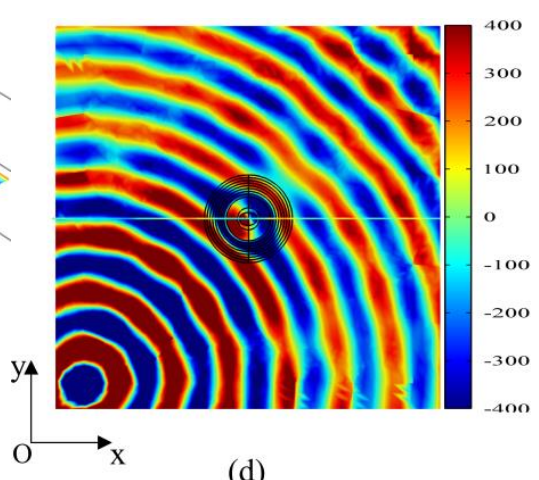

(d)

Fig. 10. Electric field distribution for the 8-layered cloak with a cylindrical wave: (a) 3D field distribution without object, (b) xoy cross-sectional field distribution without object, (c) 3D field distribution with object, (d) xoy cross-sectional field distribution with object.

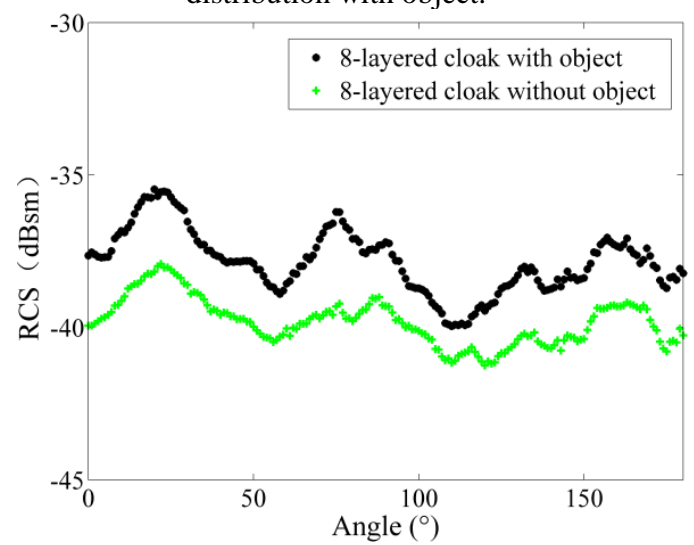

Fig. 11. Far field curves for the 8-layered cloak with and without object.

Fig. 12 shows the constitutive parameter distributions for the 8-layered invisible cloak in the region II, where $a \leq r^{\prime} \leq b$. The constitutive parameters in the region $a \leq r^{\prime} \leq b$ only had four components as $z^{\prime}=0$. Figs. 12 (a), (b), (c), and (d) depict the distributions for $\varepsilon_{x x}^{\prime}, \varepsilon_{x y}^{\prime}, \varepsilon_{y y}^{\prime}$, and $\varepsilon_{z z}^{\prime}$, respectively. The three values of $\varepsilon_{x x}^{\prime}, \varepsilon_{y y}^{\prime}$, and $\varepsilon_{z z}^{\prime}$ exhibit non-negative values, and only $\varepsilon_{x y}^{\prime}$ has some negative values. Additionally, Fig. 12 (c) can be obtained by rotating the data in Fig. 12 (a) by $90^{\circ}$. Fig. 13 shows the constitutive parameter distributions for the 8-layered invisible cloak in region III, where $b \leq r^{\prime \prime} \leq c$. The trends are similar to those in Fig. 12. 


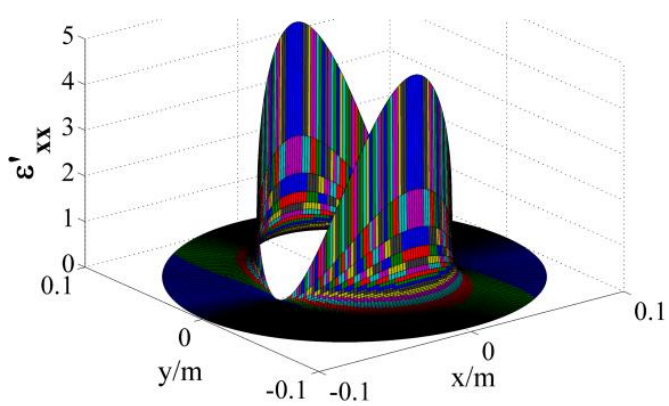

(a)

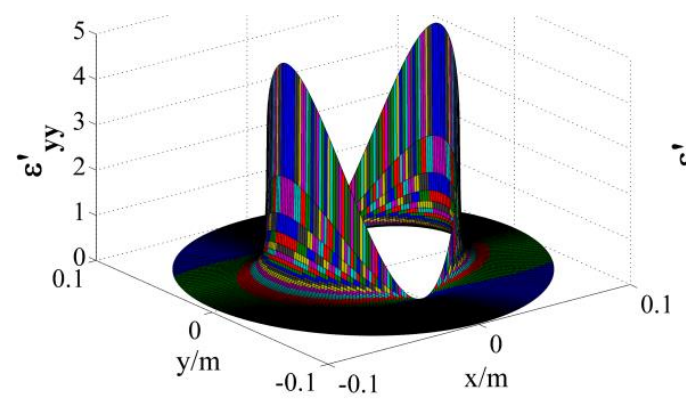

(c)

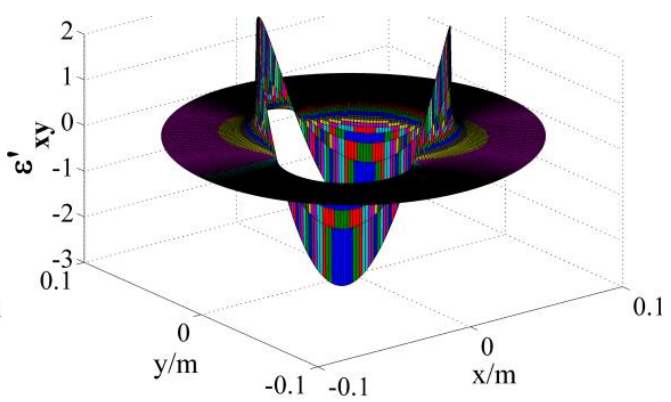

(b)

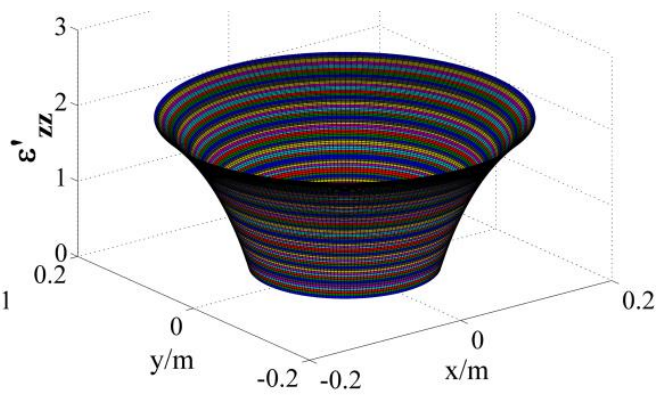

(d)

Fig. 12. Constitutive parameter distributions for the 8-layered cloak in region II $a \leq r^{\prime} \leq b$. (a) $\varepsilon_{x x}^{\prime}$, (b) $\varepsilon_{x y}^{\prime}$, (c) $\varepsilon_{y y}^{\prime}$, (d)

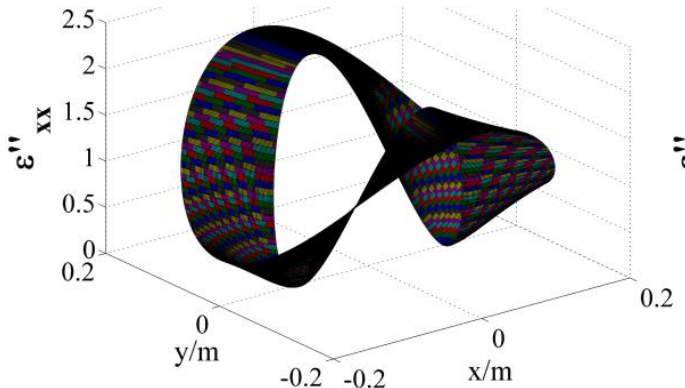

(a)

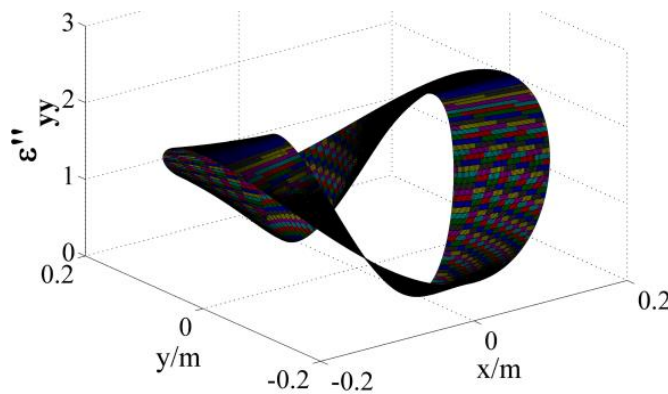

(c) $\varepsilon_{z z}^{\prime}$

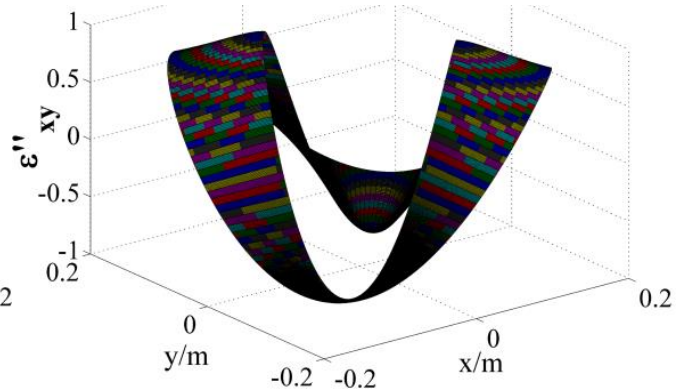

(b)

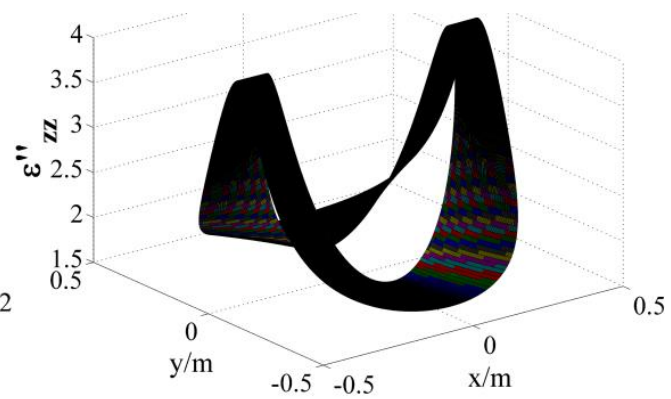

(d)

Fig. 13. Constitutive parameter distributions for the 8-layered cloak in region III $b \leq r^{\prime \prime} \leq c$. (a) $\varepsilon_{x x}^{\prime \prime}$, (b) $\varepsilon_{x y}^{\prime \prime}$, (c) $\varepsilon_{y y}^{\prime \prime}$, (d)

$$
\varepsilon_{z z}^{\prime \prime}
$$

Artificial metamaterials are usually lossy in practical applications. Thus, it is necessary to study the effects of loss in these examples. Electric and magnetic-loss tangents $(\operatorname{tg} \delta)$ are applied to measure the loss of the $E$ field in the uniform layered cloak. Fig. 14 shows the $E$ field distribution for the 8layered cloak with the different loss tangents. By comparing Figs. 14(b) and (d), it is apparent that the scattering in the latter is stronger (i.e., the color is lighter). Fig. 15 illustrates the near field curves for the different electric and magnetic-loss tangents. The two curves for $\operatorname{tg} \delta=0$ and $\operatorname{tg} \delta=0.001$ 
practically overlap. This indicates that the invisibility performance of the cloak is almost unaffected when $\operatorname{tg} \delta=0.001$. However, the power of the electromagnetic wave is strongly reduced when $\operatorname{tg} \delta \geq 0.1$.

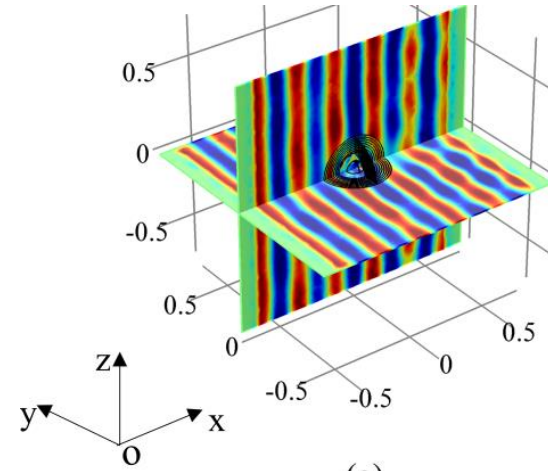

(a)

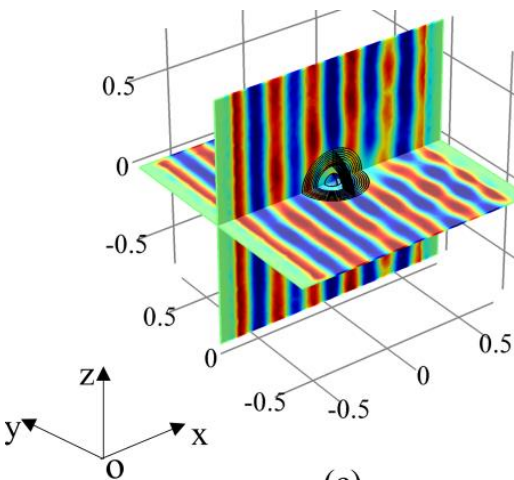

(c)

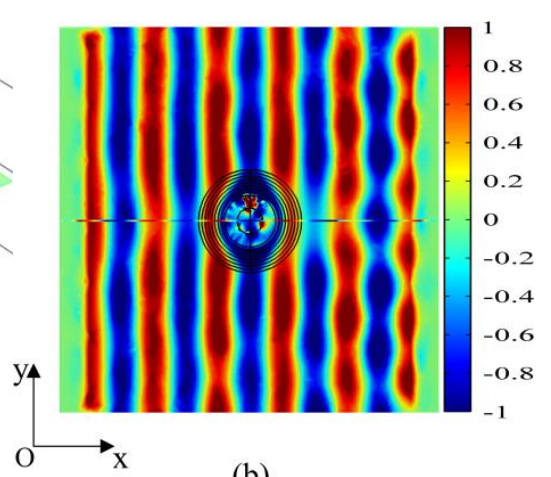

(b)

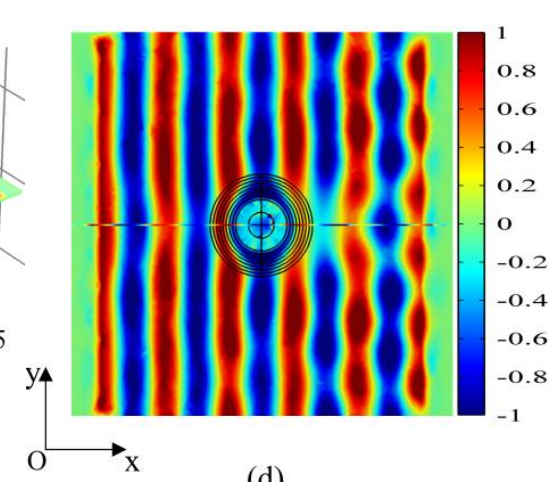

(d)

Fig. 14. Electrical field distribution for the 8-layered cloak with different loss tangents: (a) 3D field distribution when $\operatorname{tg} \delta=0.001$, (b) xoy cross-sectional field distribution when $\operatorname{tg} \delta=0.001$, (c) 3D field distribution when $\operatorname{tg} \delta=0.1$, (d) xoy cross-sectional field distribution when $\operatorname{tg} \delta=0.1$.

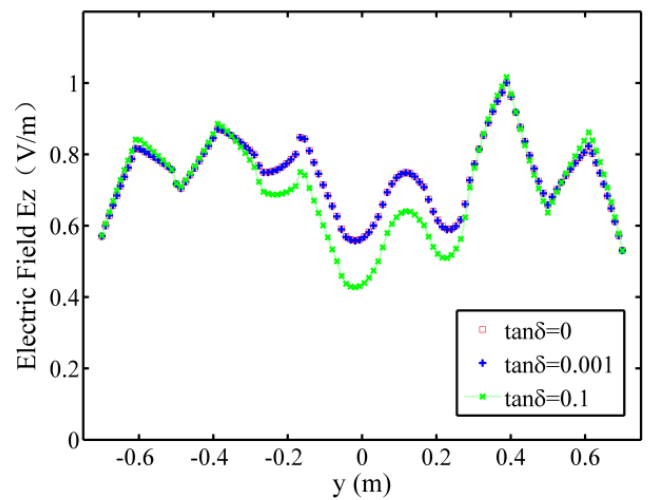

Fig. 15. Near field curves for the different electric and magnetic-loss tangents.

\section{CONCLUSION}

In this study, we proposed a uniform layered approach to design a 3D reciprocal invisible cloak and investigated its performance. To this end, we derived the constitutive parameter expressions for regions I and II of the invisible cloak component layers. In order to demonstrate the efficiency of the uniform layered cloak as a replacement for the ideal cloak, we plotted the relative permittivity and permeability curves for the different layered cloaks, simulated the electric field distribution across the material and investigate the results. Ultimately, we find that the near field of the 8-layered cloak is 
Journal of Microwaves, Optoelectronics and Electromagnetic Applications, Vol. 18, No. 2, June 2019 DOI: http://dx.doi.org/10.1590/2179-10742019v18i21551

similar to the ideal cloak. Furthermore, we computed the RCS and observed that the scattering of the 8-layered cloak in the far field was quite small. Therefore, we deduce that the ideal cloak can be replaced by a multilayered cloak to reduce difficulties in actual fabrication. Moreover, the communication ability of the layered cloak can also be demonstrated. Compared to previous designs, our design presents an effective and simplified method to obtain for the actual application of invisibility cloaks using a multilayered structure approach.

\section{ACKNOWLEDGMENT}

This work was supported by the National Natural Science Foundation of China (grant number 61731005).

\section{REFERENCES}

[1] J. B. Pendry, D. Schurig, and D. R. Smith, "Controlling electromagnetic fields," Science, vol. 312, no. 5781, pp. 1780$1782,2006$.

[2] D. Schurig, J. J. Mock, B. J. Justice, S. A. Cummer, J. B. Pendry, A. F. Starr, and D. R. Smith, "Metamaterial electromagnetic cloak at microwave frequencies," Science, vol. 314, no. 5801, pp. 977-980, 2006.

[3] H. Chen and C. T. Chan, "Transformation media that rotate electromagnetic fields," Applied Physics Letters, vol. 90, no. 24, 241105, 2007.

[4] W. X. Jiang, T. J. Cui, G. X. Yu, X. Q. Lin, Q. Cheng, and J. Y. Chin, “Arbitrarily elliptical-cylindrical invisible cloaking,” Journal of Physics D: Applied Physics, vol. 41, no. 19, 199801, 2008.

[5] D. H. Kwon and D. H. Werner, "Two-dimensional eccentric elliptic electromagnetic cloaks," Applied Physics Letters, vol. 92, no. 1, 013505, 2008.

[6] K. Yao, C. Li, and F. Li, "Electromagnetic invisibility of elliptic cylinder cloaks," Chinese Physics Letters, vol. 25, no. 5, pp. 1657-1660, 2008.

[7] H. Ma, S. B. Qu, Z. Xu, J. Q. Zhang, B. Chen, and J. F. Wang, "Material parameter equation for elliptical cylindrical cloaks," Physical Review A, vol. 77, no. 1, 013825, 2008.

[8] C. Li and F. Li, "Two-dimensional electromagnetic cloaks with arbitrary geometries," Optics Express, vol. 16, no. 17, pp. 13414-13420, 2008.

[9] J. J. Zhang, Y. Luo, H. S. Chen, and B. I. Wu, "Cloak of arbitrary shape," Journal of the Optical Society America B, vol. 25, no. 11, pp. 1776-1779, 2008.

[10] H. Ma, S. B. Qu, Z. Xu, and J. F. Wang, “Approximation approach of designing practical cloaks with arbitrary shapes," Optics Express, vol. 16, no. 20, pp. 15449-15454, 2008.

[11] G. X. Yu, T. J. Cui, and W. X. Jiang, "Design of transparent structure using metamaterial," Journal of Infrared Millimeter and Terahertz Waves, vol. 30, no. 6, pp. 633-641, 2009.

[12] M. Rahm, D. Schurig, D. A. Roberts, S. A. Cummer, D. R. Smith, and J. B. Pendry, "Design of electromagnetic cloaks and concentrators using form-invariant coordinate transformations of Maxwell's equations," Photonics and Nanostructures-Fundamentals and Applications, vol. 6, no. 1, pp. 87-95, 2008.

[13] H. Ma, S. B. Qu, Z. Xu, and J. F. Wang, “The open cloak,” Applied Physics Letters, vol. 94, no. 10, 103501, 2009.

[14] Y. Lai, H. Y. Chen, Z. Q. Zhang, and C. T. Chan, "Complementary media invisibility cloak that cloaks objects at a distance outside the cloaking shell," Physical Review Letters, vol. 102, no. 9, 093901, 2009.

[15] Y. Luo, S. Z. Zhu, L. X. He, and Y. Wang, "Arbitrary polygonal cloaks with multiple invisible regions," Journal of Modern Optics, vol. 58, no. 1, pp. 14-20, 2011.

[16] J. J. Yang, M. Huang, C. F. Yang, and J. Yu, "Reciprocal invisibility cloak based on complementary media," European Physical Journal D, vol. 61, no. 3, pp. 731-736, 2011.

[17] C. F. Yang, J. J. Yang, M. Huang, Z. Xiao, and J. H. Peng, "An external cloak with arbitrary cross section based on complementary medium and coordinate transformation," Optics Express, vol. 19, no. 2, pp. 1147-1157, 2011.

[18] Y. Urzhumov, N. Landy, T. Driscoll, D. Basov, and D. R. Smith, "Thin low-loss dielectric coatings for free-space cloaking," Optics Letters, vol. 38, no. 10, pp. 1606-1608, 2013.

[19] R. Dehbashi and M. Shahabadi, "External cylindrical invisibility cloaks with small material dynamic range," IEEE Transaction on Antennas and Propagation, vol. 62, no. 4, pp. 2187-2191, 2014.

[20] Y. Tian, C. Lu, R. F. Wang, and Z. L. Mei, “An exterior anti-cloak,” Plasmonics, Vol. 11, No. 5, 1213-1217, 2016.

[21] P. Vura, A. Rajput, and K. V. Srivastava, "Composite-shaped external cloaks with homogeneous material properties," IEEE Antennas and Wireless Propagation Letters, vol. 15, pp. 282-285, 2016.

[22] J. B. Pendry and S. A. Ramakrishna, "Focusing light using negative refraction," Journal of Physics: Comdensed Matter, vol. 15 , no. 37 , pp. 6345-6364, 2003. 